IdeAs

Idées d'Amériques

$7 \mid 2016$

Cinéma et histoire dans les Amériques

\title{
Patrick Howlett-Martin, La coopération médicale internationale de Cuba. L'altruisme récompensé
}

Paris, L'Harmattan, 2015, 207 p.

\section{Alvar De la Llosa}

\section{OpenEdition}

\section{Journals}

Electronic version

URL: https://journals.openedition.org/ideas/1418

DOI: 10.4000/ideas.1418

ISSN: 1950-5701

\section{Publisher}

Institut des Amériques

Electronic reference

Alvar De la Llosa, "Patrick Howlett-Martin, La coopération médicale internationale de Cuba. L'altruisme récompensé", IdeAs [Online], 7| 2016, Online since 27 June 2016, connection on 19 October 2022. URL: http://journals.openedition.org/ideas/1418; DOI: https://doi.org/10.4000/ideas.1418

This text was automatically generated on 19 October 2022 .

\section{(ब) $\Theta$}

Creative Commons - Attribution-NonCommercial-NoDerivatives 4.0 International - CC BY-NC-ND 4.0 https://creativecommons.org/licenses/by-nc-nd/4.0/ 


\title{
Patrick Howlett-Martin, La coopération médicale internationale de Cuba. L'altruisme récompensé
}

Paris, L'Harmattan, 2015, 207 p.

\author{
Alvar De la Llosa
}

\section{REFERENCES}

Patrick Howlett-Martin, La coopération médicale internationale de Cuba. L'altruisme récompensé, Paris, L'Harmattan, 2015, 207 p.

1 S'ouvrant sur une allocution de Fidel Castro prononcée en mai 1998 à Genève dans le cadre de la fondation de l'OMS, on pouvait craindre qu'on ne se trouve devant un énième ouvrage de propagande en faveur du régime castriste. Or, il n'en est rien, bien au contraire, l'ouvrage de Patrick Howlett-Martin est à la fois pondéré et fort bien informé. L'auteur, membre de la diplomatie française, propose un travail marqué par la richesse des chiffres et des données. La bibliographie est ample (60 pages sur 207), les sources cubaines et étasuniennes dominent, mais aussi africaines et françaises; les articles provenant de revues médicales abondent, et les sites internet sont favorisés, ce qui est à la fois garant de qualité scientifique et facilite la consultation d'une partie des sources.

2 Après des années de silence médiatique, la qualité du système d'intervention médical cubain est désormais reconnue, notamment depuis la lutte contre le virus Ébola. Cuba a été le plus grand bailleur de fonds et le plus grand fournisseur de personnel médical qualifié. Cela fut, on s'en souvient, reconnu en octobre 2014 par le secrétaire d'État américain John Kerry qui, dans un communiqué officiel, célébrait l'envoi de 165 médecins et infirmiers cubains en Afrique de l'Ouest. Toujours fin 2014, dans un éditorial inattendu de la part d'un journal qui n'a jamais eu la dent tendre avec la politique de La Havane, le New York Times («Cuba's Impressive Role on Ebola », 19 
octobre 2014) écrivait des mots qui allaient prendre tout leur sens à la mi-décembre 2014 : « This should serve as an urgent reminder to the Obama administration that the benefits of moving swiftly to restore diplomatic relations with Cuba far outweigh the drawbacks». La qualité de l'action humanitaire cubaine aurait donc, entre autres éléments, aidé à la restauration de relations rompues depuis 1961 .

Pour l'auteur, le développement spectaculaire du système médical cubain est justement dû à la carence de médecins et de spécialistes qui préférèrent émigrer au début de la Révolution plutôt que de voir leur niveau de vie baisser. La moitié des 6912 médecins abandonnent une île où les deux tiers des hôpitaux se concentrent dans la capitale, alors que la moitié de la population vit dans les campagnes. Face à ce manque et ce déséquilibre, l'État développe un système de formation rapide qui recrute de jeunes campagnards plus au fait des conditions de vie tropicale. La coopération internationale débute en 1963 en Algérie où la décolonisation a provoqué le départ des médecins français. Aujourd'hui, le personnel de santé cubain à l'étranger est supérieur à celui de toutes les nations du G-8. L'auteur explique ce phénomène unique avec une abondance de données, et par un ample panorama de l'état de la santé dans diverses régions du Tiers monde. Il compare les situations afin de mieux comprendre les raisons du déploiement de l'action médicale cubaine, et il a soin de la placer dans la réalité planétaire. L'abondance de données extraites d'une étude contrastée de sources variées (institutionnelles ou médicales) dévoile des réalités inattendues et met en relief l'absurdité de l'aide internationale trop souvent dictée, voire imposée, par la Banque mondiale ou le FMI à nombre de pays sans ressources. Si la coopération médicale cubaine est née d'une volonté d'intervention politique dans le cadre de la décolonisation, elle a su évoluer jusqu'à la professionnalisation qu'on lui reconnaît aujourd'hui, concentrant désormais $80 \%$ de son action en Amérique latine et non plus en Afrique. Dans les pays d'Amérique latine les plus démunis, l'aide médicale s'accompagne de campagnes d'alphabétisation.

4 Sans angélisme, l'auteur révèle que cette coopération est aussi devenue une source de devises pour l'État cubain puisque, hormis pour les pays les plus indigents, elle est facturée. En 2013, elle a rapporté environ 8,2 milliards de dollars, soit plus que le tourisme. En revanche, si le coût financier n'est pas évalué, on sait que le Venezuela prend en charge le transport et une partie de la logistique dans le cadre de l'organisation ALBAMed.

5 Howlett-Martin s'attarde sur l'École latino-américaine de Médecine (ELAM), sa fondation, son fonctionnement et les raisons de sa réussite, notamment l'offre d'études faite à des jeunes gens issus de milieux défavorisés et provenant de divers pays du monde. Il remarque aussi comment ce système international se heurte parfois à des mentalités paysannes très différentes d'un pays à l'autre. Commence alors un catalogue détaillé des lieux d'exercice et des spécialités déployées selon les régions (enfants de Tchernobyl, lutte contre la cécité en Amérique latine, handicapés, procédures d'urgence face aux catastrophes naturelles (ouragan Mitch, séisme à Haïti ou au Pakistan).

6 Les pages consacrées aux études de médecine aux États-Unis montrent que le coût exorbitant des études provoque une course à la spécialisation et une installation dans les beaux quartiers urbains afin de les rembourser. Cette réalité, et d'autres, permet de comprendre l'enjeu que représente la formation à Cuba d'étudiants étasuniens issus de 
communautés défavorisées qui se heurtent d'ailleurs au Trading with the Ennemy Act de 1917.

7 La pratique de la médecine est indissociable des médicaments. Face aux restrictions imposées à l'île par l'embargo étasunien, et face au coût prohibitif des médicaments produits par les grandes multinationales, l'ALBAFarma a été créé avec les partenaires économiques de l'ALBA (Alliance bolivarienne pour les peuples de notre Amérique, 2004). Les relations entre les grandes entreprises pharmaceutiques et le système bancaire international, voire les fonds de placement, provoquent une disparité du prix des médicaments, même entre les États-Unis et le Canada. Cuba développe avec ses partenaires ses productions sur des segments de marchés délaissés parce que jugés non rentables par les grandes compagnies pharmaceutiques. Se pose alors le problème des produits génériques et de la législation adoptée lors de l'Uruguay Round de 1986-1994. L'auteur offre une vision globale des rapports entre les institutions internationales, l'industrie pharmaceutique et les enjeux au niveau mondial, cela afin de mieux comprendre le créneau dans lequel le système médical cubain internationalisé se glisse. La projection cubaine dépasse le cadre latino-américain bien au-delà de l'ALBA. À partir de 2014, ALBAMed régule et enregistre des médicaments produits à l'échelle régionale, pendant qu'ALBAFarma les achète et les distribue.

8 Les diverses actions déployées en Haïti, en Amérique centrale, en Équateur, en Bolivie, au Brésil, au Venezuela, et même en Argentine et en Uruguay, pays réputés développés, sont passées en revue. L'action médicale cubaine en Afrique, au Moyen-Orient, en Asie et dans le Pacifique fait l'objet d'un long développement, tant les lieux et les actions sont nombreux et spécifiques. L'accueil est parfois très hostile notamment au Brésil, voire en Bolivie, au Venezuela et en Amérique centrale, où les médecins locaux voient d'un très mauvais œil l'arrivée de nouvelles pratiques. Dans ce contexte, l'auteur attire aussi l'attention sur les diverses raisons qui facilitent la fuite des cerveaux médicaux qui affecte le Tiers monde. Dans le cas cubain, l'adoption aux États-Unis du Cuban Medical Professional Parole Program a permis en 5 ans de débaucher entre 1600 et 8000 médecins cubains engagés principalement au Venezuela, en Bolivie et en Colombie. Mais leur intégration hors de l'île est rendue difficile, comme celle des étudiants de l'ELAM quand ils reviennent dans leurs pays. Le niveau de formation est jugé insuffisant. Moyen de les tenir à l'écart ou réalité ? Au-delà de positions idéologiques anti-communistes, les médecins cubains sont aussi victimes de préjugés racistes contre les Noirs.

Le revers de la médaille existe évidemment. Cuba a formé un nombre élevé de médecins généralistes aux dépens de spécialisations médicales. L'action extérieure est souhaitée par les autorités qui peuvent ainsi rentabiliser un nombre sans doute trop important de médecins dans l'île. Cette générosité à l'égard de l'extérieur entraîne des critiques. Les départs provoqueraient une perte de qualité des soins et une pénurie de spécialistes. Au-delà des consultations, les prestations hospitalières laisseraient à désirer. Les équipements de certains services seraient vétustes. De plus, la réalité médicale cubaine n'échappe pas aux contrecoups des bouleversements que l'île subit depuis 1991. Le salaire d'un médecin dépasse rarement les 64 euros, alors qu'il atteint beaucoup plus dans les secteurs liés au tourisme et aux activités privées qui en découle. Dans les années à venir, un départ massif de médecins est à craindre. Le tourisme médical n'est pas encouragé par les autorités car il se concentre surtout sur la chirurgie esthétique et le dentaire. La continuation de la formation est mise en danger par des États africains 
qui ne versent pas à leurs étudiants la partie de la bourse qui leur correspond, et par les difficultés à faire valider leurs diplômes au retour. Par ailleurs, l'éclat de l'action cubaine se verrait aujourd'hui terni par la spectaculaire progression de l'action médicale chinoise, notamment en Afrique. Un millier de médecins chinois travaillent dans 42 pays. Au cours des 10 dernières années, Pékin a construit 30 hôpitaux en Afrique, et en a modernisé des dizaines. La Chine possède des moyens financiers et techniques sans commune mesure avec l'indigence cubaine, au-delà du fait que la majeure partie des financements en matière de santé destinés aux pays en développement provient de fonds étasuniens et européens. Mais arrivent-ils à destination? Par ailleurs, les récents développements de l'actualité en Amérique latine, autant que la baisse du cours du pétrole, remettent en cause la prolongation du financement vénézuélien. Finalement, les bienfaits du système de santé cubain ont contribué à rapprocher l'île des caractéristiques européennes, parmi elles, le vieillissement de la population. Comme le signale l'auteur, au moment où les dirigeants cubains entreprennent des réformes inévitables, les acquis sociaux et l'altruisme médical ne seront-ils pas emportés par la transformation des structures économiques?

10 Ce livre s'inscrit dans le vaste cadre des relations nord-sud, il n'offre pas une vision idyllique mais un juste regard sur un travail difficile rendu éprouvant dans bien des cas par les catastrophes naturelles comme par l'opposition des élites locales, mais toujours gratifié par l'accueil des populations locales démunies.

Par l'abondance des données et le sérieux de ses sources, l'ouvrage de Patrick HOWLETT-MARTIN se révèle indispensable à tous ceux qui veulent comprendre les raisons de la survie du régime cubain, le rayonnement international de ce petit pays et sa capacité à développer une action diplomatique atypique, celle-là même que le 19 avril 2009 à Port-of-Spain, Barack Obama qualifiait, en lui rendant hommage, de softdiplomacy.

\section{AUTHORS}

\section{ALVAR DE LA LLOSA}

Professeur en civilisation latino-américaine, Université Lyon 2 